\title{
Alternations in DNA gyrase genes in low-level fluoroquinolone-resistant Moraxella catarrhalis strains isolated in Poland
}

This article was published in the following Dove Press journal: Infection and Drug Resistance

\author{
Katarzyna Król-Turmińska \\ Alina Olender \\ Department of Medical Microbiology, \\ Medical University of Lublin, Lublin, \\ Poland
}

Purpose: The purpose of this study was to investigate the molecular mechanisms of fluoroquinolone resistance in Moraxella catarrhalis clinical strains isolated in Lublin, Poland.

Materials and methods: A total of 150 non-duplicate clinical strains of $M$. catarrhalis were obtained from individuals with signs of upper respiratory tract infection. Bacterial identification was corroborated on the basis of phenotypic and biochemical characteristics as well as with the use of molecular tests. The antimicrobial susceptibility of $M$. catarrhalis isolates was determined using the disk diffusion method and Etest. Mutations in the gyrase ( $g y r A$ and $g y r B)$ and topoisomerase ( parC and parE) genes were determined by polymerase chain reaction and sequencing. Results: It was observed that $16.7 \%$ of the studied isolates were drug resistant. Resistance to tetracycline was detected for $12 \%$ of the strains. Resistance to nalidixic acid, moxifloxacin, and levofloxacin was exhibited by $2.7 \%$ of the strains; $1.3 \%$ of the strains were resistant to trimethoprim/sulfamethoxazole and $0.7 \%$ to erythromycin. Minimum inhibitory concentration values of the four strains demonstrating fluoroquinolone resistance were: $6-12 \mathrm{mg} / \mathrm{L}$ for nalidixic acid, $1-1.5 \mathrm{mg} / \mathrm{L}$ for levofloxacin, $1 \mathrm{mg} / \mathrm{L}$ for moxifloxacin, and $0.25-0.5 \mathrm{mg} / \mathrm{L}$ for ciprofloxacin. The research resulted in the detection of mutations in 4 strains, in gyrase gyr $A$ and $g y r B$ genes. In gyrA gene, there occurred mutation $\mathrm{G} 412 \mathrm{C}$ as well as four silent transition mutations. Within gyrB gene, there occurred mutation, substitution A1481G, as well as two identical silent mutations.

Conclusion: Our findings reveal that resistance to fluoroquinolones in M. catarrhalis is connected with amino acid substitutions in $\operatorname{gyr} A$ and $\operatorname{gyr} B$ genes. To our knowledge, this work is the first description of fluoroquinolone-resistant clinical strains of $M$. catarrhalis with described mutations in gyrA and gyrB genes isolated in Poland and in Europe.

Keywords: Moraxella catarrhalis, drug resistance, quinolones, respiratory tract infections

\section{Introduction}

Moraxella catarrhalis is an aerobic Gram-negative diplococcus that is frequently isolated from the human respiratory tract. For many years, $M$. catarrhalis has been considered a harmless commensal microorganism, but recent research proves the important pathogenic character of the bacterium. It is mainly responsible for the infections of upper and lower respiratory tract including acute otitis media in children and exacerbation of chronic obstructive pulmonary disease in adults. Less frequently, it may lead to severe infections of different locations which are heavily dependent on the host's condition. ${ }^{1,2}$ Despite the widely described inherent resistance of the bacteria to vancomycin, clindamycin, and trimethoprim and their extremely common resistance to penicillins, resulting from the production of specific $\beta$-lactamases, most clinical
Correspondence: Katarzyna

Król-Turmińska

Department of Medical Microbiology, Medical University of Lublin, Chodźki I, 20-093 Lublin, Poland

Tel +48 8I 4486400

Email katarzynakrolturminska@umlub.p 
isolates are susceptible to most of the antibiotics commonly used in the treatment of respiratory infections. Due to that, infections caused by $M$. catarrhalis are usually treated with a combination of penicillin and $\beta$-lactamase inhibitors. Moreover, most of macrolides, tetracyclines, quinolones, and trimethoprim/sulfamethoxazole are usually highly effective against these bacteria with good therapeutic effects. ${ }^{3-5}$

Fluoroquinolones have a broad spectrum of action and high therapeutic safety. Due to their good penetration into respiratory tract and laryngeal tissues, they are readily used in the treatment of infections of these areas. Newer generations of drugs in this class (eg, levofloxacin and moxifloxacin) are effective against Gram-positive, Gram-negative, aerobic, and anaerobic bacteria. They are also proven to be effective against some atypical bacteria. Fluoroquinolones inhibit DNA synthesis by targeting two essential topoisomerases in bacterial cell. The first one, DNA gyrase, an enzyme found exclusively in bacteria, uses the energy of ATP hydrolysis to introduce negative supercoils into DNA, which is essential for chromosome condensation, replication, and promoting transcription initiation. The second enzyme, topoisomerase IV, resolves interlinked daughter chromosomes after DNA replication and also relaxes positive supercoils of DNA strands. Both enzymes are composed of two pairs of subunits. The A subunit of DNA gyrase is encoded by the gyr $A$ gene, whereas the $\mathrm{B}$ subunit is encoded by $\operatorname{gyr} B$ gene. The $A$ and B topoisomerase IV subunits are encoded, respectively, by parE and parC genes. The resistance of bacteria to fluoroquinolones can result from three mechanisms. In Gram-negative bacteria, the most common of these is the presence of mutations (amino acid substitutions in quinolone resistance-determining region [QRDR]) within the genes encoding the A and B subunits of gyrase and topoisomerase IV. In addition, fluoroquinolones can be actively pumped out of the cell via efflux mechanism, and some species of bacteria also produce Qnr proteins that form stable complexes with gyrase and topoisomerase IV inhibiting the activity of these enzymes as a result. ${ }^{6}$

Despite the generally high antibiotic sensitivity of $M$. catarrhalis, sporadic reports show a slow gradual growth of the number of drug-resistant strains. Fluoroquinolone resistance in $M$. catarhalis is still a very rare phenomenon; however, it has been reported by several countries. These include, among others, the USA,${ }^{7}$ Europe,${ }^{8}$ India, $,{ }^{9}, 10$ Taiwan, ${ }^{11}$ Thailand, ${ }^{10}$ and Japan. ${ }^{12}$ To date, the exact mechanism of fluoroquinolone resistance in $M$. catarrhalis has not been fully clarified, and there exist only a few reports dealing with this subject. ${ }^{12,13}$ Thus, the purpose of this study was to investigate the molecular mechanisms of fluoroquinolone resistance in M. catarrhalis clinical strains isolated in Lublin, Poland.

\section{Materials and methods Bacterial strains and culture conditions}

The bacterial strains were obtained from the upper respiratory tract specimens (nasal or pharyngeal swabs) collected from individuals with signs of infection of this area. The only inclusion criteria for patients were primary care doctor's recommendation for microbiological testing and positive cultures for $M$. catarrhalis in the respiratory sample. One hundred fifty non-duplicate clinical strains of $M$. catarrhalis were used in this study. Bacterial cultures were grown routinely for $24 \mathrm{~h}$ at $37^{\circ} \mathrm{C}$ on Columbia agar (Becton, Dickinson and Company, Franklin Lakes, NJ, USA) in an atmosphere comprising $95 \%$ air and $5 \% \mathrm{CO}_{2}$.

\section{Identification}

The fact that the obtained isolates belonged to $M$. catarrhalis species was corroborated on the basis of phenotypic and biochemical characteristics as well as with the use of molecular tests. Bacterial colonies on blood agar with phenotype characteristics for $M$. catarrhalis, that is, round, convex, shiny, opaque, about $2 \mathrm{~mm}$ in diameter, light gray, of equal thickness, not hemolytic, and which can be pushed along the surface of the agar (hockey disk test), were transferred to a fresh medium to obtain pure cultures. Colonies of Gramnegative, catalase- and oxidase-positive diplococci were additionally identified using API ${ }^{\circledR} \mathrm{NH}$ identification strips (bioMerieux, Marcy l'Etoile, France).

For a definitive identification of the examined strains, polymerase chain reaction (PCR) for marker gene $\operatorname{cop} B$ was performed. DNA extraction was performed using a commercial kit (QIAamp DNA Mini Kit, Qiagen, Hilden, Germany). The nucleotide primer sequences to amplify this gene were previously described by Verhaegh et $\mathrm{al}^{14}$ and are given in Table 1. A standard PCR protocol was used. It comprised 1 cycle of initial denaturation at $94^{\circ} \mathrm{C}$ for $3 \mathrm{~min}$, denaturation at $94^{\circ} \mathrm{C}$ for $40 \mathrm{~s}$, annealing at a temperature of $55^{\circ} \mathrm{C}$ for $1 \mathrm{~min}$, and extension at a temperature of $72^{\circ} \mathrm{C}$ for $1 \mathrm{~min}$, each for 35 cycles. Thermocycling was conducted with the use of LabCycler Gradient thermocycler (SensoQuest, Gottingen, Germany). PCR products were visualized by electrophoresis on $2 \%$ agarose gels (Basica LE, ABO, Gdańsk, Poland) and stained with ethidium bromide (ICN Biomedicals, Aurora, OH, USA). M. catarrhalis ATCC 25238 was used as a positive control. 
Table I PCR primers for $\operatorname{copB}$ and quinolone resistance-determining region (QRDR) amplification in Moraxella catarrhalis

\begin{tabular}{lllll}
\hline Name & Sequence $\left(\mathbf{5}^{\prime} \rightarrow \mathbf{3}^{\prime}\right)$ & Temperature $\left({ }^{\circ} \mathbf{C}\right)$ & Amplicon size (bp) & Reference \\
\hline copB forward & GGCGTGCGTGTTGACCGTTTTG & 58.6 & 564 \\
copB reverse & GTTTGGCAGGCGATAGGCGACAT & 58.8 & 370 \\
gyrA forward & TGCGTGATGGACTTAAGCCT & 51.8 & \\
gyrA reverse & GGCAACACGCTTGGCATTTT & 51.8 & 460 \\
gyrB forward & GTGGTTGAGACCGCCATGCA & 55.9 & \\
gyrB reverse & CATCCGCATCGGTCATGATG & 53.8 & 390 \\
parC forward & GCATCGTCTATGCCATGAGT & 51.8 & \\
parC reverse & CGGCGATGCCTGTCGTGCCA & 60.0 & 340 \\
parE forward & GTCAGTCAGCGGGTGGATCA & 55.9 & \\
parE reverse & CCTGCATCAATACGATACAA & 47.7 &
\end{tabular}

\section{Antimicrobial susceptibility testing}

Cefinase disks (MAST ID Graso Biotech, Poland) were used to investigate $\beta$-lactamase production. Antimicrobial susceptibility testing was conducted using the Kirby-Bauer disk diffusion method on MH-F agar (bioMerieux) in compliance with the European Committee on Antimicrobial Susceptibility Testing (EUCAST) guidelines and interpreted in accordance with the "breakpoint tables for interpretation of minimum inhibitory concentrations (MICs) and zone diameters" specified by EUCAST for each year. ${ }^{15}$ The study involved the use of antibiotic disks in appropriate concentrations from Becton, Dickinson and Company. Furthermore, fluoroquinolone susceptibility of the four fluoroquinoloneresistant M. catarrhalis strains was examined on MH-F agar using an Etest (bioMerieux) to estimate the MIC value. $M$. catarrhalis ATCC 25238 strain was used as a control to ascertain the accuracy of the performed identification.

\section{PCR and DNA sequencing of fluoroquinolone-resistant genes}

In order to detect mutations in the QRDR regions, the following gene fragments of fluoroquinolone-resistant strains were analyzed: nt 172-447 region of gyrA, nt 1135-1527 region of gyrB , nt 187-507 region of parC, and nt 1321-1590 region of parE. These gene fragments underwent PCR reaction. Then, the obtained products of these reactions underwent sequencing. DNA was extracted with the use of a commercial kit (QIAamp DNA Mini Kit) in compliance with producer's guidelines. Primer sequences and the expected quantities of the reaction products are presented in Table 1. The PCR reaction protocol was identical to the one performed for $c o p B$ gene, except for the fact that 30 cycles were used in the reaction process. The products of PCR reaction were sequenced with the use of the Sanger method (Genomed, Warsaw,
Poland). The obtained results underwent a comparative analysis involving a gene sequence of wild-type $M$. catarrhalis strain BBH18 (GenBank Accession No CP002005) with the use of BLAST software (software available online: www. ncbi.nlm.nih.gov). Simultaneous comparison of multiple sequences was conducted using MEGA 6 software (Molecular Evolutionary Genetics Analysis software version 6.0.).

\section{Ethics approval and informed consent}

The research protocol was approved by the Ethics Committee of the Medical University of Lublin (number KE-0254/155/2015). The study was conducted in the Department of Medical Microbiology of the Medical University of Lublin (Poland) in the years 2013-2015. All patients gave their written informed consent to the use of the isolated strains for the purpose of this study. STATISTICA 13.0 software (StatSoft, Cracow, Poland) was used to set up and maintain a database.

\section{Results}

\section{Characteristics of the studied group}

A total of 150 non-duplicate $M$. catarrhalis isolates were collected from 62 males and 88 females. One hundred twentyseven $(84.7 \%)$ isolates were obtained from nasal swabs and 23 (15.3\%) from pharyngeal swabs. The average age of individuals who provided materials for isolates was $6.23 \pm 10.52$ years, and the age range of the patients was from 3 months to 68 years. Most of the M. catarrhalis isolates were recovered from children younger than 4 years of age $(141 ; 94 \%)$.

\section{Antimicrobial susceptibility}

About $98.7 \%(n=148)$ of the studied strains produced $\beta$-lactamases (positive cefinase test), whereas 1.3\% $(n=2)$ did not produce these enzymes. The study assessed the 
sensitivity of $M$. catarrhalis isolates to the following groups of antimicrobial substances: penicillins (amoxicillin/clavulanate $20 / 10 \mu \mathrm{g} / \mathrm{disk}$ ), cephalosporins (cefuroxime $30 \mu \mathrm{g} / \mathrm{disk}$ ), fluoroquinolones (nalidixic acid $30 \mu \mathrm{g} / \mathrm{disk}$, ciprofloxacin $5 \mu \mathrm{g} /$ disk, moxifloxacin $5 \mu \mathrm{g} /$ disk, levofloxacin $5 \mu \mathrm{g} / \mathrm{disk}$ ), macrolides (erythromycin $15 \mu \mathrm{g} /$ disk), tetracyclines (tetracycline $30 \mu \mathrm{g} / \mathrm{disk}$ ), and trimethoprim/sulfamethoxazole (trimethoprim/sulfamethoxazole 1.25-23.75 $\mu \mathrm{g} / \mathrm{disk}$ ). The studied isolates were classified based on breakpoint values of growth inhibition zones determined by EUCAST into two categories: resistant or susceptible, and in case of tetracycline also into intermediate category. Twenty-five $(16.7 \%)$ of the studied isolates were drug resistant. Almost all drug-resistant strains were isolated from children under the age of $4(93.9 \%)$ years and from materials obtained from nasal swabs $(84.8 \%)$. In general, 8 drug-resistant strains were isolated in 2013, 6 in 2014, and 11 in 2015. Resistance to tetracycline was demonstrated by 18 (12\%) of the strains, whereas a decreased sensitivity to this substance was reported for 22 (14.7\%) of the strains. Among fluoroquinolone drugs, resistance to nalidixic acid, moxifloxacin, and levofloxacin was demonstrated by $4(2.7 \%)$ strains. In case of the rest of the antimicrobial substances, $2(1.3 \%)$ strains were resistant to trimethoprim/sulfamethoxazole and $1(0.7 \%)$ was resistant to erythromycin. Resistance to amoxicillin/clavulanate, ciprofloxacin, and cefuroxime was not detected (Table 2). MIC values of the 4 strains demonstrating fluoroquinolone resistance are presented in Figure
Table 2 Drug susceptibility of Moraxella catarrhalis isolates

\begin{tabular}{ll}
\hline Antibiotic & Number of resistant strains \\
\hline Tetracycline & $12 \%(n=18)$ \\
Nalidixic acid & $2.7 \%(n=4)$ \\
Moxifloxacin & $2.7 \%(n=4)$ \\
Levofloxacin & $2.7 \%(n=4)$ \\
Ciprofloxacin & 0 \\
Erythromycin & $0.7 \%(n=1)$ \\
Trimethoprim/sulfamethoxazole & $1.3 \%(n=2)$ \\
Cefuroxime & 0 \\
Amoxicillin/clavulanate & 0 \\
\hline
\end{tabular}

1. These were: $6-12 \mathrm{mg} / \mathrm{L}$ for nalidixic acid, $1-1.5 \mathrm{mg} / \mathrm{L}$ for levofloxacin, $1 \mathrm{mg} / \mathrm{L}$ for moxifloxacin, and $0.25-0.5 \mathrm{mg} / \mathrm{L}$ for ciprofloxacin (Figure 1).

\section{Sequencing}

The work adopts a chronological numbering of strains with regard to the order of their isolation. The research resulted in the detection of mutations in 4 strains, in gyrase gyr $\mathrm{A}$ and $\operatorname{gyr} B$ genes (Figure 2). Mutations did not occur in topoisomerase parC and parE genes. Mutations in gyrA gene occurred in the genetic material of 1 strain (no 52). A single missense mutation $\mathrm{G} 412 \mathrm{C}$ resulting in the substitution of Gln138 (CAG) for Glu138 (GAG) was observed in case of this strain. The analyzed gene fragment of strain 52 also featured four silent transition mutations: C234T,

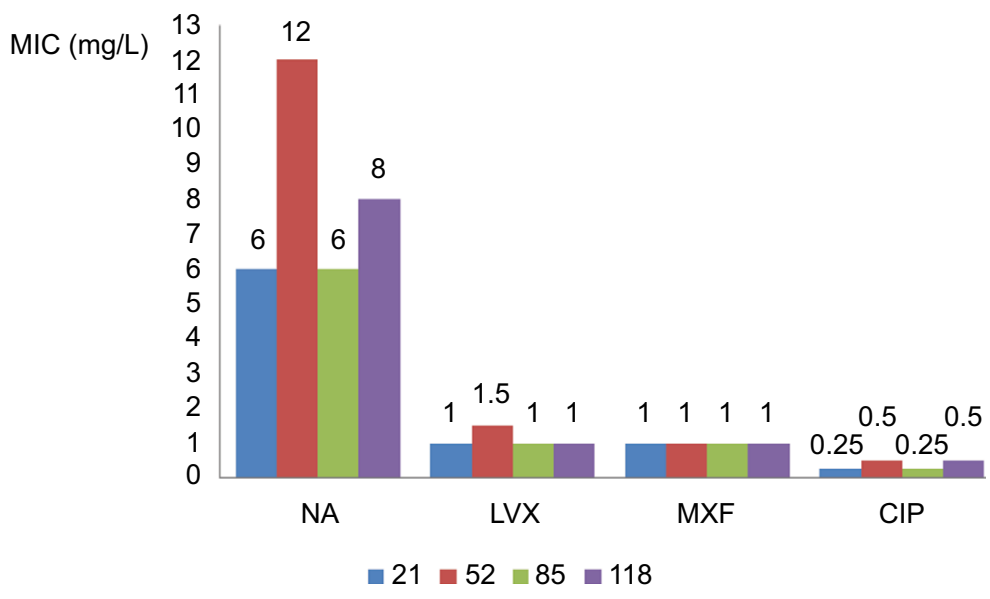

Figure I MIC values of examined fluoroquinolones.

Note: $21,52,85$, and 118 are strain numbers.

Abbreviations: NA, nalidixic acid; LVX, levofloxacin; MXF, moxifloxacin; CIP, ciprofloxacin; MIC, minimum inhibitory concentration. 


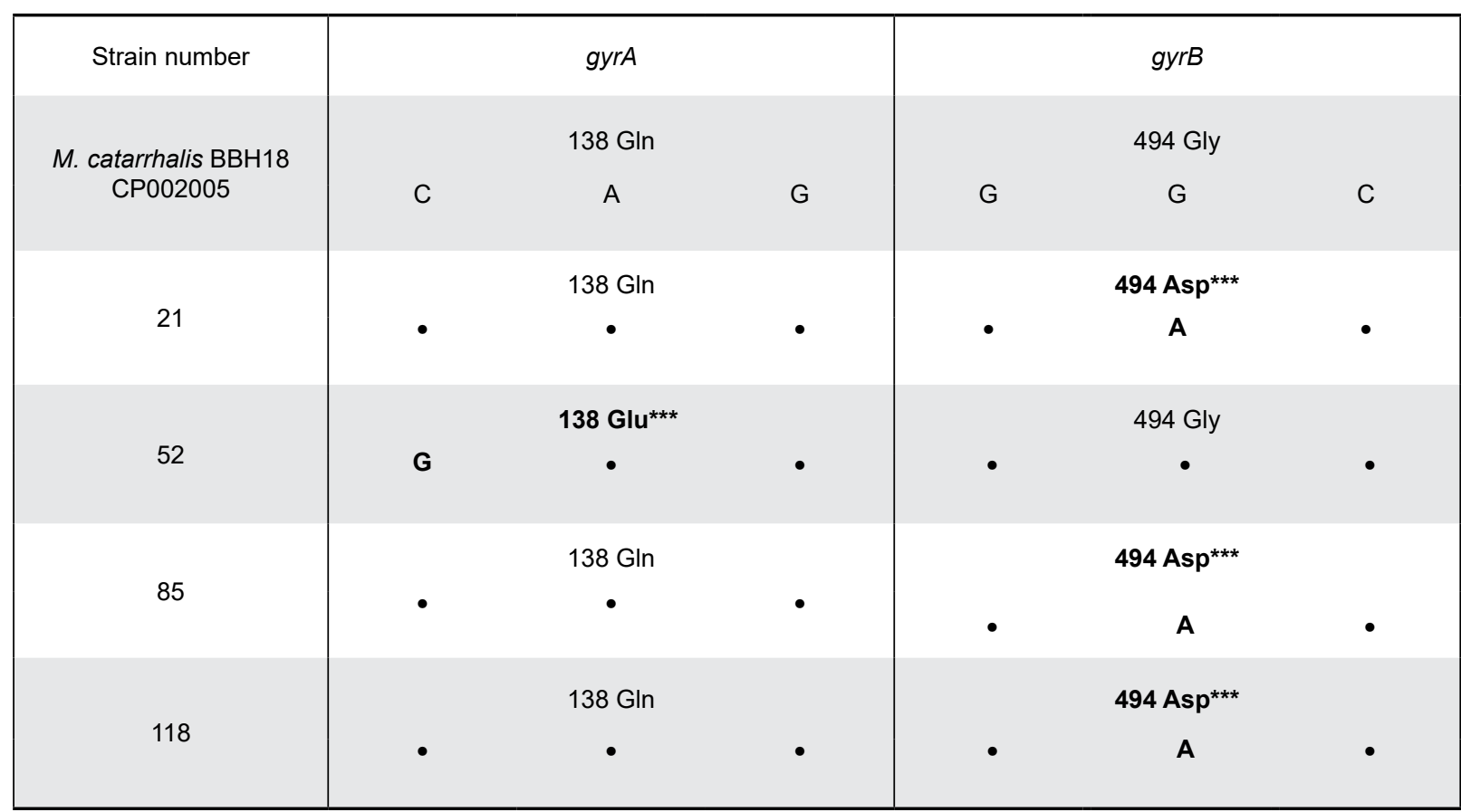

Figure 2 Nonsynonymous mutations in bacterial DNA gyrase genes, gyrA and gyrB, in fluoroquinolone-resistant Moraxella catarrhalis strains. Notes: The dots indicate the absence of a mutation in the tested sequence. The bold texts indicate the place where the mutation occurred in the tested sequence. ***indicates missense mutation (which results in a protein in which one amino acid is substituted for another).

C243T, T306C, and C336T. Within gyrB gene of strains 21, 85 , and 118 , there occurred an identical missense mutation, substitution A1481G, which resulted in the substitution of Gly494 (GGC) for Asp494 (GAC), as well as two identical silent mutations in case of each strain: transition A1446G and transversion A1404T.

\section{Discussion}

Antimicrobial agents from the fluoroquinolone family are widely and willingly applied in the therapy of multiple infections. However, the increased prevalence of fluoroquinoloneresistant strains of various bacterial species has recently become a serious risk in several places around the world. ${ }^{12,16-18}$ In this work, most of the drug-resistant clinical isolates of $M$. catarrhalis were obtained from children. Fluoroquinolone antibiotics are not commonly used in this age group owing to the risk of side effects. At the same time, this age group is characterized by the highest rate of $M$. catarrhalis colonization. It might suggest that fluoroquinolone-resistant strains isolated from children can be strains acquired from adults.

Previous works demonstrated that fluoroquinolone resistance in Gram-negative bacteria is most often conditioned by mutations in gyr $A$ and/or parC genes, whereas mutations in gyrB and parE genes result in low-level resistance phenotypewise. Additionally, there is an increase of fluoroquinolone resistance in case of many QRDR mutations. ${ }^{6,19}$ Other mechanisms were described in the group of Gram-negative bacteria as well. These, however, were not connected with the changes in the QRDR region, which condition this type of drug resistance. Molecular basis of Gram-negative bacterial resistance to fluoroquinolones has already been described both for cocci and for bacilli such as, among others, Neisseria gonorrhoeae, N. meningitidis, Escherichia coli, Salmonella enterica, Shigella spp., Campylobacter jejuni, Haemophilus influenzae. ${ }^{20-27}$ The present work describes 4 strains resistant to fluoroquinolones which possessed mutations in gyr $A$ and $g y r B$ genes within their genome. In these strains, the occurrence of mutations was connected to low-level resistance to levofloxacin and moxifloxacin as well as with resistance to nalidixic acid. All 4 isolates were sensitive to ciprofloxacin. The occurrence of mutations in $g y r A$ gene resulted in a slightly higher levofloxacin and nalidixic acid MIC values in comparison to the strains with mutations only in $\operatorname{gyr} B$ gene. There exist only a few studies describing the resistance mechanisms of $M$. catarrhalis to fluoroquinolones in world literature, and all of them were conducted in Japan. The first work describing mutations in the QRDR region of $M$. catarrhalis was conducted by Yamada and Saito. ${ }^{12}$ It involved the analysis of $5 \mathrm{M}$. catarrhalis strains exhibiting a decreased sensitivity to fluoroquinolones (ciprofloxacin 
MIC of $0.5 \mathrm{mg} / \mathrm{L}$ and levofloxacin MIC of $1 \mathrm{mg} / \mathrm{L})$. All 5 studied strains featured a gyrA gene mutation - C239T (Thr $80>$ Ile). The work corroborated that a Thr-to-Ile substitution at amino acid 80 (T80I) in gyr $A$ gene had a considerable influence on the lowering of strains' sensitivity to fluoroquinolones, especially levofloxacin. During the study, there were no changes detected in parC gene. The same authors and associates described 6 novel mutations in their latest work. These mutations occurred in gyrA (Asp 84> Tyr, Thr594dup, and Ala722dup), gyrB (Glu 479> Lys and Asp 439> Asn), and parE (Gln 395> Arg), all of which are involved in $M$. catarrhalis resistance to fluoroquinolones. ${ }^{27}$ Iwata et al, ${ }^{13}$ in a work also conducted in Japan, analyzed a single clinical $M$. catarrhalis strain resistant to macrolides and fluoroquinolones (levofloxacin MIC of $4 \mathrm{mg} / \mathrm{L}$ ). The researchers discovered the presence of mutation C239T (Thr 80> Ile) in gyrA gene and A1481G (Asp 494> Gly) in gyrB gene. There was also a single silent mutation in par $C$ gene, whereas there were no mutations detected in parE gene. The mutation in $g y r B$ gene that was described in their study was identical to the one observed in the research conducted by the authors of the following work, whereas the Glu 138> Gln mutation detected in gyr $A$ gene has not been described so far. In conclusion, our findings reveal that the resistance to fluoroquinolones in $M$. catarrhalis is connected with amino acid substitutions in $g y r A$ and $g y r B$ genes. The acquisition of mutations in QRDR region of $M$. catarrhalis is most probably a direct cause of the occurrence of fluoroquinolone resistance in this species, and the mutations in gyrA gene condition a higher level of resistance. However, other mechanisms conditioning this type of resistance, that is, efflux mechanism or the production of Qnr proteins, were not researched in this study and they require further verification.

\section{Conclusion}

To our knowledge, this work is the first description of fluoroquinolone-resistant clinical strains of $M$. catarrhalis with described mutations in $g y r A$ and $g y r B$ genes isolated in Poland and in Europe. The work was conducted within a small area. Thus, there exists a strong need to expand and continue the studies across the whole country. Due to the occurrence of fluoroquinolone resistance in $M$. catarrhalis as well as the resistance to other antibiotics that has been observed increasingly more often, drug susceptibility testing of this pathogen to antibiotics should be implemented as a routine procedure as a part of the diagnostic process. The possibility to conduct epidemiological surveillance of the occurrence of drug-resistant strains of this species in Poland and other countries would be worth taking into consideration as well. It is also vital to draw the attention to the increasing number of drug-resistant $M$. catarrhalis strains in Europe and worldwide and to monitor this alarming phenomenon.

\section{Disclosure}

The authors report no conflicts of interest in this work.

\section{References}

1. Karalus R, Campagnaria A. Moraxella catarrhalis: a review of an important human mucosal pathogen. Microbes Infec. 2000;2:547-559.

2. Aebi C. Moraxella catarrhalis - pathogen or commensal? Adv Exp Med Biol. 2011;697:107-116.

3. Khan MA, Northwood JB, Levy F, et al. Bro\{beta\}-lactamase and antibiotic resistances in a global cross-sectional study of Moraxella catarrhalis from children and adults. J Antimicrob Chemother. 2010;65:91-97.

4. McGregor K, Chang BJ, Mee BJ, et al. Moraxella catarrhalis: clinical significance, antimicrobial susceptibility and BRO betalactamases. Eur J Clin Microbiol Infect Dis. 1998;17:219-234.

5. Shaikh SB, Ahmed Z, Arsalan SA, et al. Prevalence and resistance pattern of Moraxella catarrhalis in community-acquired lower respiratory tract infections. Infect Drug Resist. 2015;8:263-267.

6. Fàbrega $\mathrm{A}$, Madurga S, Giralt E, et al. Mechanism of action of and resistance to quinolones. Microb Biotechnol. 2009;2:40-61.

7. DiPersio JR, Jones RN, Barrett T, et al. Fluoroquinolone-resistant Moraxella catarrhalis in a patient with pneumonia: report from the SENTRY Antimicrobial Surveillance Program (1998). Diagn Microbiol Infect Dis. 1998;32:131-135.

8. Flamm RK, Sader HS, Farrell DJ, et al. Macrolide and tetracycline resistance among Moraxella catarrhalis isolates from 2009 to 2011. Diagn Microbiol Infect Dis. 2012;74:198-200.

9. Gupta N, Arora S, Kundra S. Moraxella catarrhalis as a respiratory pathogen. Indian J Pathol Microbiol. 2011;54:769-771.

10. Torumkuney D, Chaiwarith R, Reechaipichitkul W, et al. Results from the Survey of Antibiotic Resistance (SOAR) 2012-14 in Thailand, India, South Korea and Singapore. J Antimicrob Chemother. 2016;71:3-19.

11. Hsu SF, Lin YT, Chen TL, et al. Antimicrobial resistance of Moraxella catarrhalis isolates in Taiwan. J Microbiol Immunol Infect. 2012;45:134-140.

12. Yamada K, Saito R. Molecular analysis of low-level fluoroquinolone resistance in clinical isolates of Moraxella catarrhalis. JMed Microbiol. 2014;63:1066-1070.

13. Iwata S, Sato Y, Toyonaga Y, et al. Genetic analysis of a pediatric clinical isolate of Moraxella catarrhalis with resistance to macrolides and quinolones. $J$ Infect Chemother. 2015;21:308-311.

14. Verhaegh SJC, Streefland A, Dewnarain JK, et al. Age-related genotypic and phenotypic differences in Moraxella catarrhalis isolates from children and adults presenting with respiratory disease in 2001-2002. Microbiol. 2008;154:1178-1184.

15. Clinical breakpoints-bacteria v $3.1 ; 4.0 ; 5.0$. Available from: http:// www.eucast.org/ast_of_bacteria/previous_versions_of_documents/. Accessed September 15, 2017.

16. Yaghoubi S, Ranjbar R, Soltan Dallal MM, et al. The frequency of mutations in quinolone resistance-determining regions and plasmidmediated quinolone resistance in Shigella isolates recovered from pediatric patients in Tehran, Iran: an overlooked problem. Microb Drug Resist. Epub 2017 Nov 17.

17. Lee H, Ahn S, Hwang NY, et al. Limited effect of later-generation fluoroquinolones in the treatment of ofloxacin-resistant and moxifloxacin-susceptible multidrug-resistant tuberculosis. Antimicrob Agents Chemother. 2018;62(2). pii: e01784-17. 
18. Shun-Mei E, Zeng JM, Yuan H, et al. Sub-inhibitory concentrations of fluoroquinolones increase conjugation frequency. Microb Pathog. 2018;114:57-62.

19. Deguchi T, Yasuda M, Nakano M, et al. Quinolone-resistant Neisseria gonorrhoeae: correlation of alterations in the GyrA subunit of DNA gyrase and the ParC subunit of topoisomerase IV with antimicrobial susceptibility profiles. Antimicrob Agents Chemother. 1996;40:1020-1023.

20. Malehmir S, Ranjbar R, Harzandi N. The molecular study of antibiotic resistance to quinolones in Salmonella enterica strains isolated in Tehran, Iran. Open Microbiol J. 2017; 21:189-194.

21. Kang M, Wei B, Choi SW, et al. Molecular characterization of fluoroquinolone resistance mechanisms of Campylobacter isolates from duck meats. J Food Prot. 2017;80:2056-2059.

22. Kondo H, Ito S, Hatazaki K, et al. GyrA and/or ParC alterations of Haemophilus influenzae strains isolated from the urethra of men with acute urethritis. $J$ Infect Chemother. 2018;24:232-235.
23. Sartori L, Fernandes MR, Ienne S, et al. Draft genome sequences of two fluoroquinolone-resistant CTX-M-15-producing Escherichia coli ST90 (ST23 complex) isolated from a calf and a dairy cow in South America. J Glob Antimicrob Resist. 2017;11:145-147.

24. Jiang FX, Lan Q, Le WJ, Su XH. Antimicrobial susceptibility of Neisseria gonorrhoeae isolates from Hefei (2014-2015): genetic characteristics of antimicrobial resistance. BMC Infect Dis. 2017;17:366.

25. Castanheira M, Deshpande LM, Jones RN, et al. Evaluation of quinolone resistance-determining region mutations and efflux pump expression in Neisseria meningitidis resistant to fluoroquinolones. Diagn Microbiol Infect Dis. 2012;72:263-166.

26. Garoff L, Yadav K, Hughes D. Increased expression of Qnr is sufficient to confer clinical resistance to ciprofloxacin in Escherichia coli. $J$ Antimicrob Chemother. 2018;73:348-352

27. Yamada K, Saito R, Muto S, et al. Molecular characterization of fluoroquinolone-resistant Moraxella catarrhalis variants generated in vitro by stepwise selection. Antimicrob Agents Chemother. 2017:22;61(10). pii: e01336-17.
Infection and Drug Resistance

\section{Publish your work in this journal}

Infection and Drug Resistance is an international, peer-reviewed openaccess journal that focuses on the optimal treatment of infection (bacterial, fungal and viral) and the development and institution of preventive strategies to minimize the development and spread of resistance. The journal is specifically concerned with the epidemiology of antibiotic

\section{Dovepress}

resistance and the mechanisms of resistance development and diffusion in both hospitals and the community. The manuscript management system is completely online and includes a very quick and fair peerreview system, which is all easy to use. Visit http://www.dovepress.com/ testimonials.php to read real quotes from published authors. 